\title{
Probing and dressing magnetic impurities in a superconductor
}

\author{
K. Akkaravarawong, ${ }^{1}$ J. I. Väyrynen, ${ }^{2}$ J. D. Sau, ${ }^{3}$ E. A. Demler, ${ }^{4}$ L. I. Glazman,${ }^{5}$ and N. Y. Yao ${ }^{1,6}$ \\ ${ }^{1}$ Department of Physics, University of California, Berkeley, California 94720, USA \\ ${ }^{2}$ Microsoft Quantum, Microsoft Station Q, University of California, Santa Barbara, California 93106-6105 USA \\ ${ }^{3}$ Condensed Matter Theory Center, Department of Physics and Joint Quantum Institute, University of Maryland, College Park, \\ Maryland 20742, USA \\ ${ }^{4}$ Department of Physics, Harvard University, Cambridge, Massachusetts 02138, USA \\ ${ }^{5}$ Department of Physics, Yale University, New Haven, Connecticut 06520, USA \\ ${ }^{6}$ Materials Science Division, Lawrence Berkeley National Laboratory, Berkeley, California 94720, USA
}

(Received 3 June 2019; revised manuscript received 30 September 2019; published 11 November 2019; corrected 1 October 2020)

\begin{abstract}
We propose a method to probe and control the interactions between an ensemble of magnetic impurities in a superconductor via microwave radiation. Our method relies upon the presence of subgap Yu-Shiba-Rusinov (YSR) states associated with the impurities. Depending on the sign of the detuning, radiation generates either a ferro- or antiferromagnetic contribution to the exchange interaction. This contribution can bias the statistics of the random exchange constants stemming from the Ruderman-Kittel-Kasuya-Yosida (RKKY) interaction. Moreover, by measuring the microwave response at the YSR resonance, one gains information about the magnetic order of the impurities. To this end, we estimate the absorption coefficient as well as the achievable strength of the microwave-induced YSR interactions using off-resonant radiation. The ability to utilize microwave fields to both probe and control impurity spins in a superconducting host may open new paths to studying metallic spin glasses.
\end{abstract}

DOI: 10.1103/PhysRevResearch.1.033091

\section{INTRODUCTION}

The nature of interactions between magnetic impurities embedded in a metallic host gives rise to an intriguing state of matter: a spin glass [1]. The Ruderman-Kittel-Kasuya-Yosida (RKKY) exchange interaction between the impurities is carried by itinerant electrons and alternates in sign, depending on the inter-impurity separation [2-4]. The random position of the impurities with respect to each other results in a randomsign exchange interaction, frustrating the magnetic order in a system of localized spins. The efforts to understand the resulting low-temperature spin glass phase and the corresponding phase transition have led to the introduction of several important concepts in condensed-matter physics, including the Edwards-Anderson [5] and functional [6] order parameters. Moreover, these efforts have also motivated an ever-expanding tool set of quantum control techniques aimed at directly controlling the interactions between magnetic impurities.

Remarkably, even the simplest spin glass model introduced by Sherrington and Kirkpatrick [7] in direct analogy to the Curie-Weiss model of a ferromagnet turns out to be extremely rich and, unlike the Curie-Weiss model, not amenable to a straightforward mean-field theory treatment [8,9]. The frustration of the magnetic moments manifests itself in both the

Published by the American Physical Society under the terms of the Creative Commons Attribution 4.0 International license. Further distribution of this work must maintain attribution to the author(s) and the published article's title, journal citation, and DOI. thermodynamic and electron transport properties of a normal metal with a magnetic element dissolved in it. Starting with magnetic susceptibility measurements on AuFe alloys [10], there are a substantial number of such studies performed on bulk samples [11,12]. With the development of mesoscopic systems, electron transport through mesoscale-sized alloys also received their fair share of attention; for example, the remanence of the resistance (i.e., its dependence on the cooling protocol) of a mesoscopic AgMn device was investigated in (a)

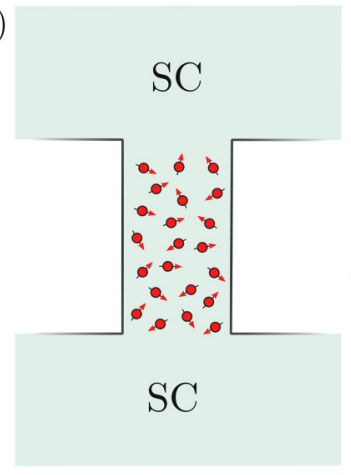

(b)
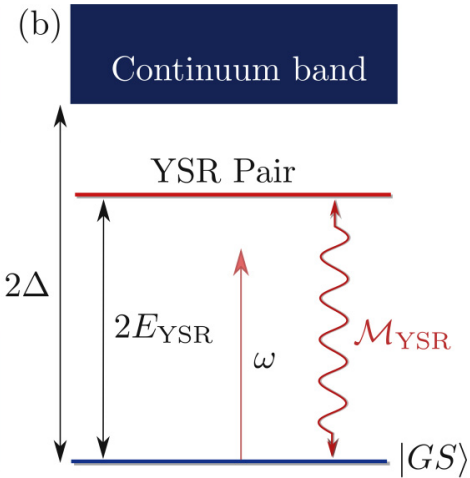

FIG. 1. (a) Schematic of an S-s-S junction consisting of two large superconducting banks connected by a narrow constriction, with the arrows representing magnetic impurities. (b) Energy levels of the YSR states associated with two magnetic impurities in a superconductor. The radiation matrix element $\mathcal{M}_{\text {YSR }}$ depends on the mutual spin orientation of the impurities (i.e., it vanishes for parallel magnetic moments), resulting in a spin-dependent AC Stark shift that translates to an effective, microwave-induced, spin-spin interaction. 
[13], while quantum interference effects in the conductance of CuMn and AgMn were studied respectively in Refs. [14,15].

In this paper, we explore a method for investigating mesoscopic spin glasses using techniques recently perfected in the development of superconducting qubit technologies [16]. In particular, we consider the possibility of utilizing microwave radiation to directly probe and possibly control the many-body state of an ensemble of magnetic moments embedded in a thin superconducting bridge (Fig. 1).

Much as in a normal metal, magnetic impurities in a superconductor also exhibit random-sign RKKY interactions. This RKKY interaction is hardly modified by superconductivity, so long as the typical distance $d$ between the impurities is shorter than the superconducting coherence length $\xi$. For impurities separated by larger distances, the interaction is instead antiferromagnetic and dominated by a virtual process involving Yu-Shiba-Rusinov (YSR) states [17]; however, at such distances, the interactions are typically weak since they decay exponentially with $d / \xi$. Herein lies the intuition behind our approach: To utilize microwave driving to enhance the virtual hybridization between the superconducting condensate and the YSR states.

With respect to such microwave excitation, there are two main differences between normal-metal and superconducting hosts. The first is that there exists a gap $\Delta$ in the spectrum of excitations in a superconductor. In the absence of a magnetic field and impurities, a conventional $s$-wave superconductor, such as aluminum, possesses time-reversal symmetry. As a result, the gap is "hard": At low temperatures there is a frequency threshold, $\omega_{\text {th }}=2 \Delta / \hbar$, for the absorption of electromagnetic radiation. The second difference is that a magnetic impurity in a superconductor creates a localized YSR state with energy $E_{Y S R}$ within the gap [18-26]. A single YSR state may host no more than one quasiparticle, and therefore cannot facilitate absorption of a photon by exciting a Cooper pair from the condensate [27]. However, a pair of YSR states separated by distances $\lesssim \xi_{\text {YSR }}$ creates a discrete-energy state for an electron pair where $\xi_{\mathrm{YSR}}=\xi \sqrt{\Delta /\left(\Delta-E_{\mathrm{YSR}}\right)}$ is the characteristic length scale of a YSR state. To this end, at low temperatures, the subgap absorption results from a process in which a microwave photon transfers a Cooper pair from the condensate onto the pair of YSR states, leading to an absorption line centered at $\omega=2 E_{\mathrm{YSR}} / \hbar$.

Crucially, the magnitude of this absorption by a YSR pair depends on the mutual orientation of the magnetic moments. For moments oriented in parallel, the associated pair of YSR states cannot accept a singlet Cooper pair (for simplicity, we assume that there is no spin-orbit coupling). Thus, the absorption is maximized for antiparallel moments and varies as $F\left[\mathbf{S}\left(\mathbf{R}_{1}\right), \mathbf{S}\left(\mathbf{R}_{2}\right)\right]=1-\hat{\mathbf{S}}_{1} \cdot \hat{\mathbf{S}}_{2}$, where $\hat{\mathbf{S}}_{1,2}=\mathbf{S}_{1,2} / S$ are the unit vectors indicating the orientation of the magnetic moments. Therefore, the subgap absorption coefficient provides information regarding ferromagnetic order at the scale, $\left|\mathbf{R}_{1}-\mathbf{R}_{2}\right| \lesssim \xi_{Y S R}$. The absorption line width and its detailed shape depend on the inevitable spread of the contact exchange interaction [28-31] and the overlap between the YSR states [24,32-34].

For an ensemble of moments with density $n \gtrsim \xi^{-3}$, the many-body ferromagnetic order can be deduced from
$\bar{F}(\{\mathbf{S}(\mathbf{r})\})=\left\langle\sum_{i \neq j} K\left(\left|\mathbf{R}_{i}-\mathbf{R}_{j}\right|\right) F\left[\mathbf{S}\left(\mathbf{R}_{i}\right), \mathbf{S}\left(\mathbf{R}_{j}\right)\right]\right\rangle / N$, where $\langle\ldots\rangle$ denotes averaging over YSR state energies, $K(r) \sim$ $\Theta\left(\xi_{\mathrm{YSR}}-r\right) \quad(\Theta$ is the Heaviside step function), $N \sim$ $n^{2} \xi^{3} \mathcal{V}(w / \xi)^{3-D}$ is a normalization factor, and $\mathcal{V}$ is the volume of the sample which we think of as either a wire $(D=1)$ or as a film $(D=2)$ with transverse dimension $w \ll \xi$. This quantity is related to the dissipative part of the conductivity integrated over the absorption line:

$$
\int_{0}^{2 \Delta / \hbar} d \omega \sigma(\omega)=\bar{F}(\{\mathbf{S}(\mathbf{r})\}) \Sigma, \quad \Sigma \sim \sigma_{n} \frac{n^{2}}{v^{2} \Delta} \frac{1 / n \xi^{2}}{l+1 / n \xi^{2}}
$$

where $n$ is the impurity concentration, $\sigma_{n}$ and $v$ are, respectively, the normal-state conductivity and the density of states at the Fermi level, and $l$ is the electron elastic mean free path; note that the last factor in Eq. (1) extrapolates between the regimes of long and short mean free paths.

As aforementioned, in the absence of microwaves, there are two components of the inter-impurity interaction carried by virtual excitations of the itinerant electrons. The first (and dominant at $d \lesssim \xi$ ) one comes from the continuum of Bogoliubov quasiparticles and is responsible for the conventional indirect exchange coupling, i.e., RKKY interaction in normal metals and its counterpart in superconductors. The second one owes to the discrete YSR states and is specific to superconductors. Borrowing the idea of "off-resonant dressing" from quantum optics [35], we note that off-resonant microwave radiation creates an additional channel for virtual transitions of Cooper pairs onto a pair of YSR states. For sufficiently strong drives, the corresponding amplitude may successfully compete with the one existing in the absence of radiation [17], while the conventional RKKY component is only weakly affected, so long as the radiation remains far detuned from the gap edge. The sign of the effective interaction induced by the off-resonant drive depends on the sign of the detuning, $\omega-2 E_{\mathrm{YSR}}$, and is ferromagnetic for $\omega-2 E_{\mathrm{YSR}}>0$.

One can estimate the strength of the microwave-induced interaction, $J_{\mathrm{YSR}}^{\text {ind }}$, relative to the RKKY component $J_{\mathrm{RKKY}}$ as

$$
\frac{\left\langle J_{\mathrm{YSR}}^{\text {ind }}\right\rangle}{\left\langle J_{\mathrm{RKKY}}\right\rangle} \sim \frac{\Delta}{2 E_{\mathrm{YSR}}-\omega}\left(\frac{d}{\xi}\right)^{3}\left(\frac{e \mathcal{E} \xi}{\omega}\right)^{2},
$$

where $\mathcal{E}$ is the electric field of the microwave. In order for superconductivity to remain intact, the last factor here must be small; to avoid resonant absorption, the denominator of the first factor must exceed the YSR absorption line width. Together, these two conditions set a limit for the strength of the "dressed" interaction. Intriguingly, in a dilute system, at $d \sim \xi$, the dressed interaction may compete with the conventional RKKY component opening up the possibility of studying the de Almeida-Thouless line [9] in a spin glass.

\section{MODEL}

Our starting point is the BCS Hamiltonian of an $s$-wave superconductor with magnetic impurities. The Bogoliubov-de Gennes (BdG) Hamiltonian takes the form

$$
\mathcal{H}=\epsilon_{p} \tau_{z}+\Delta \tau_{x}-\sum_{i} J_{i} \mathbf{S}_{i} \cdot \boldsymbol{\sigma} \delta\left(\mathbf{r}-\mathbf{R}_{i}\right),
$$


where $\epsilon_{p}=-\frac{1}{2 m} \nabla^{2}-\mu$ is the kinetic energy (we set $\hbar=$ 1), $\mu$ the chemical potential, and $\Delta$ the superconducting order parameter. The Hamiltonian $H=\int d \mathbf{r} \Psi^{\dagger} \mathcal{H} \Psi / 2$ is written in conventional Nambu spinor notation, where $\Psi=$ $\left[\psi_{\uparrow}, \psi_{\downarrow}, \psi_{\downarrow}^{\dagger},-\psi_{\uparrow}^{\dagger}\right]^{T}$ and $\tau(\sigma)$ are Pauli matrices acting on the particle-hole (spin) space. The last term in the Hamiltonian represents the contact interaction between electrons and the impurity spins, where $J_{i}$ characterizes the coupling strength and $\mathbf{S}_{i}$ is the spin of the $i$ th impurity located at position $\mathbf{R}_{i}$.

The energy of the subgap YSR bound state localized around impurity $i$ is $E_{\mathrm{YSR}}^{i}=\Delta\left(1-\alpha_{i}^{2}\right) /\left(1+\alpha_{i}^{2}\right)$ [18-20], where $\alpha_{i}=v \pi J_{i} S / 2$ is a dimensionless exchange coupling. We treat the impurity spins classically assuming they have equal magnitudes $S$ but in general different orientations. The four-component eigenspinors of the BdG Hamiltonian $\mathcal{H}$ corresponding to the subgap YSR states are given by

$$
\Phi_{i, \mathbf{S}_{i}}^{+}(\mathbf{r})=\frac{1}{\mathcal{N}_{i}} \frac{e^{-\left|\sin 2 \delta_{i}\right| r_{i} / \xi}}{k_{F} r_{i}} U\left(\hat{\mathbf{S}}_{i}\right)\left(\begin{array}{c}
\sin \left(k_{F} r_{i}+\delta_{i}\right)|\uparrow\rangle \\
\sin \left(k_{F} r_{i}-\delta_{i}\right)|\uparrow\rangle
\end{array}\right),
$$

and $\Phi_{i, \mathbf{S}_{i}}^{-}=C T \Phi_{i, \mathbf{S}_{i}}^{+}$where a particle (+) and hole (-) states $\Phi_{i, \mathbf{S}_{i}}^{ \pm}$have energies $\pm E_{\mathrm{YSR}}^{i}$ and are related by the antiunitary symmetry transformation $C T=\tau_{y} \sigma_{y} K$ where $K$ denotes complex conjugation; $\mathcal{N}_{i}^{2}=2 \pi v \Delta \alpha_{i} /\left(1+\alpha_{i}^{2}\right)$ is a normalization factor, $r_{i}=\left|\mathbf{r}-\mathbf{R}_{i}\right|$ the relative distance from the impurity, $\delta_{i}=\tan ^{-1}\left(\alpha_{i}\right)$ the phase shift, $|\uparrow\rangle$ is the +1 eigenstate of $\sigma_{z}$, and $U\left(\hat{\mathbf{S}}_{i}\right)$ is a unitary rotation operator aligning the quantization axis of the Nambu spinor $\Psi$ with the direction of the impurity spin. As we are interested in the physics resulting from low-energy microwave excitation, we will project the electron field operator onto these subgap YSR states,

$$
\Psi(\mathbf{r})=\sum_{i}\left[\Phi_{i, \mathbf{S}_{i}}^{+}(\mathbf{r}) \gamma_{i}+\Phi_{i, \mathbf{S}_{i}}^{-}(\mathbf{r}) \gamma_{i}^{\dagger}\right],
$$

where $\gamma_{i}$ is the annihilation operator of a YSR state located at the $i$ th impurity. The projected Hamiltonian then becomes $H=\sum_{i} E_{i} \gamma_{i}^{\dagger} \gamma_{i}+\sum_{i, j}\left(\mathcal{M}_{i j} \gamma_{i}^{\dagger} \gamma_{j}^{\dagger}+\mathcal{M}_{i j}^{\prime} \gamma_{i}^{\dagger} \gamma_{j}+\right.$ H.c. $)$. We focus on densities $n \ll 1 /\left(\xi \lambda_{F}^{2}\right)$ where we can ignore the hopping term $\gamma_{i}^{\dagger} \gamma_{j}$. The calculation of the radiation matrix element then reduces to an effectively two-impurity problem. At higher densities, one enters the phase of gapless superconductivity, see Ref. [32] and the references therein.

\section{RADIATION MATRIX ELEMENT}

We now turn to calculating the matrix element $\mathcal{M}_{\text {YSR }}$, corresponding to radiation-assisted YSR-pair creation. When the system is coupled to a weak microwave field, the vector potential $\tilde{\mathbf{A}}$ enters as $\frac{\hbar}{i} \boldsymbol{\nabla} \rightarrow \frac{\hbar}{i} \boldsymbol{\nabla}+\frac{e}{c} \tilde{\mathbf{A}}$ and the superconducting order parameter is generally both complex and spatially dependent, i.e., $\Delta(\mathbf{r})=|\Delta| e^{i \theta(\mathbf{r})}$. We choose to work in the London gauge where the order parameter is real and the integral of the new vector potential, $\mathbf{A}=\tilde{\mathbf{A}}-\frac{\hbar c}{e} \boldsymbol{\nabla} \theta(\mathbf{r})$, yields a gauge-invariant phase difference that gives rise to the supercurrent in a superconductor.

The electromagnetic perturbation to the $\mathrm{BdG}$ Hamiltonian is given by $H_{\mathrm{EM}}=\int d^{3} \mathbf{r}(e / 2) \Psi^{\dagger}(\mathbf{A} \cdot \mathbf{v}+\mathbf{v} \cdot \mathbf{A}) \Psi$, where $\mathbf{v}$ is the velocity operator. Using Eq. (5), the projected perturbation Hamiltonian takes the form $H_{\mathrm{EM}} \approx \mathcal{M}_{\mathrm{YSR}}^{1,2} \gamma_{1}^{\dagger} \gamma_{2}^{\dagger}+$
H.c., where $\mathcal{M}_{\text {YSR }}^{1,2}=\int d^{3} \mathbf{r} \mathbf{J}_{1,2} \cdot \mathbf{A}$. We assume a negligible thermal population of YSR states and thereby ignore hopping terms $\gamma_{1}^{\dagger} \gamma_{2}$. The current density $\mathbf{J}_{1,2}$ is given by

$$
\mathbf{J}_{1,2}(\mathbf{r})=\frac{e \hbar}{2 m i}\left\{\Phi_{1, \mathbf{S}_{1}}^{+}(\mathbf{r}) \nabla \Phi_{2, \mathbf{S}_{2}}^{-}(\mathbf{r})-\Phi_{2, \mathbf{S}_{2}}^{+}(\mathbf{r}) \nabla \Phi_{1, \mathbf{S}_{1}}^{-}(\mathbf{r})\right\} .
$$

Since the relevant microwave frequencies $(\omega \leqslant 2 \Delta)$ correspond to wavelengths significantly longer than both the superconducting coherence length and the characteristic YSR length scale, $\mathbf{A}$ can be treated as position independent. Moreover, since the integration domain contains all of space, the integral of the antisymmetric portion of the integrand vanishes. The integrand can therefore be symmetrized,

$$
\mathcal{M}_{\text {YSR }}^{1,2}=\frac{1}{2}(\mathbf{A} \cdot \hat{\mathbf{R}}) \hat{\mathbf{R}} \cdot \int d^{3} \mathbf{r}\left[\mathbf{J}_{1,2}(\mathbf{r})+\mathbf{J}_{1,2}(-\mathbf{r})\right],
$$

where we have also used the fact that the current density, Eq. (6), is rotationally symmetric around the axis connecting the two impurities, $\mathbf{R}=\mathbf{R}_{2}-\mathbf{R}_{1}$ and $\hat{\mathbf{R}}=\mathbf{R} /|\mathbf{R}|$, and therefore only the component of the vector potential that is parallel to this axis contributes to absorption.

Owing to the rotational symmetry, the integral is effectively two-dimensional and can be done in elliptical coordinates [27], $r_{+}=\left(r_{1}+r_{2}\right) / 2, r_{-}=r_{1}-r_{2}$, where the integral measure becomes $\int d^{3} \mathbf{r} \rightarrow 2 \pi \int_{R / 2}^{\infty} d r_{+} \int_{-R}^{R} d r_{-}\left\{r_{+}^{2}-\frac{r_{-}^{2}}{4}\right\}$. In these new coordinates, $r_{+}^{2}-r_{-}^{2} / 4=r_{1} r_{2}$, exactly canceling out the power-law decay of the YSR wave function.

For two identical impurities, the current density respects an additional reflection symmetry about the plane perpendicular to and bisecting $\mathbf{R}$. This symmetry would imply that the integral in Eq. (7) vanishes. Thus, a nonzero radiation matrix element requires the breaking of this reflection symmetry. In practice [30], this is always the case as one invariably observes fluctuations in the exchange coupling strengths, suggesting that the reflection symmetry is naturally broken by disorder effects. Therefore, we assume hereafter that the impurities are not identical, $\alpha_{1} \neq \alpha_{2}$.

After a straightforward but tedious calculation, the general expression of the matrix element can be obtained. Assuming that || $\sin 2 \delta_{1}|-| \sin 2 \delta_{2}|| \ll \xi / R$, the matrix element can be expanded to first order in the coupling difference, $\left|\alpha_{1}-\alpha_{2}\right|$, and $1 /\left(k_{F} R\right)$ as [36]

$$
\begin{aligned}
\left|\mathcal{M}_{\mathrm{YSR}}^{1,2}\right| \approx & \Delta \sqrt{F\left(\mathbf{S}_{1}, \mathbf{S}_{2}\right)} \frac{|e \overrightarrow{\mathcal{E}} \cdot \hat{\mathbf{R}}| \xi\left|\sin k_{F} R\right|}{h \omega} \frac{k_{F} \xi}{} \\
& \times e^{-R / \xi_{\mathrm{YSR}}}\left|\alpha_{1}-\alpha_{2}\right| \frac{2 \sqrt{2} \alpha^{3}}{\left(1+\alpha^{2}\right)^{3}},
\end{aligned}
$$

where $\overrightarrow{\mathcal{E}}=-\partial \mathbf{A} / \partial t$ is the electric field of the applied microwaves and $\alpha=\left(\alpha_{1}+\alpha_{2}\right) / 2$, the average coupling strength of the two impurities. One important and intriguing observation: Due to the longer intrinsic YSR length scale $\xi_{\mathrm{YSR}}=$ $\xi /|\sin 2 \delta|$ as well as the absence of a power-law decay in the matrix element, the corresponding microwave-induced interaction has a significantly longer range than both the RKKY interaction and the bare YSR interaction [17] (in the absence of microwaves). 


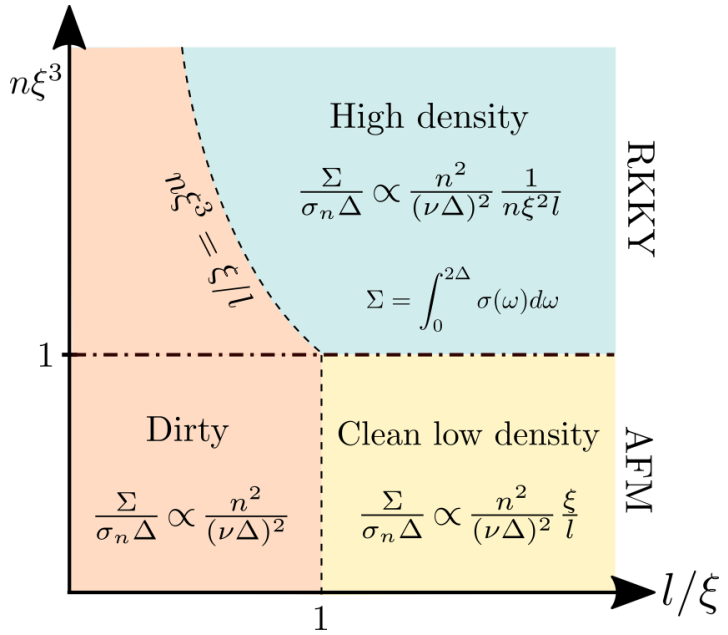

FIG. 2. Schematic cross-over diagram of the integrated sub-gap conductivity $\Sigma$ as a function of the elastic mean free path $l$ and the density of magnetic impurities $n$. In the clean and dense limit (upper right), the YSR states are strongly hybridized and $\Sigma \propto n$ [32]. At lower densities $\left(n \xi^{3} \ll 1\right)$, the hybridization is negligible and the absorption requires two magnetic impurities within $\xi$ of each other, $\Sigma \propto n^{2}$ (bottom right). In the dirty case, $l \ll$ $\min \left(\xi, 1 / n \xi^{2}\right)$, the dependence of $\Sigma$ on $n$ is similarly quadratic (left). In the dense regime, $n \xi^{3} \gg 1$ (top), the interactions between the magnetic impurities are dominated by RKKY mechanism (favoring a spin glass phase), while at low density, $n \xi^{3} \ll 1$ (bottom), the exchange interaction is exponentially weak and antiferromagnetic (AFM).

\section{EXPERIMENTAL IMPLEMENTATION}

In this section, we propose an experimental implementation based upon superconducting circuits, which enables one to utilize microwave fields to both probe the spin state of the impurities as well as control their effective interactions. Since our proposed microwave-dressed interactions require both a superconducting background and a supercurrent, a natural setup is an S-s-S junction created from two large superconducting leads linked by a constriction (Fig. 1); such a setup has previously been used in experiments to probe and control Andreev bound states [37], see also [38-40].

An oscillating bias potential, $V \cos \omega t$, can then be applied to the leads, to create a time-dependent supercurrent $j \propto$ $\frac{\hbar e}{m} \nabla \theta$ governed by the Josephson relation $\partial_{t} \theta=\frac{2 e V}{\hbar} \sin \omega t$, where $\theta$ is the gauge-invariant phase difference between the leads and $\omega$ is the microwave frequency.

To probe the many-body state of the impurities, we propose to apply a resonant microwave drive so that the ordering can be inferred from the integrated dissipative part of the subgap conductivity, $\int_{0}^{2 \Delta} \sigma(\omega) d \omega$. The dissipative conductivity $\sigma$ is related to the energy absorption rate $\omega \Gamma=\sigma \mathcal{E}^{2} / 2$, where $\Gamma$ is the transition rate obtained by plugging Eq. (8) into Fermi's golden rule. At low temperatures, the initial state consists of unoccupied YSR states. The integrated subgap conductivity depends on the average distance between impurities and the elastic mean free path $l$. It can be analyzed in various limits, which are detailed below and summarized in Fig. 2.
In the low-density limit $n \xi_{\text {YSR }}^{3} \lesssim 1$, the YSR states are well localized so that the hybridization-induced energy splitting of the YSR states is negligible compared to $\Delta$. The subgap conductivity can be written as a sum of pair-creating transitions,

$$
\sigma(\omega)=\frac{2}{\mathcal{V} \mathcal{E}^{2}} \sum_{i>j} 2 \pi \omega\left|\mathcal{M}_{\mathrm{YSR}}^{i, j}\right|^{2} \delta\left(\omega-E_{\mathrm{YSR}}^{i}-E_{\mathrm{YSR}}^{j}\right),
$$

where $\mathcal{M}_{\mathrm{YSR}}^{i, j}$ is the radiation-assisted matrix element to create a pair of YSR quasiparticles on the $i$ th and $j$ th impurity. Assuming a uniform distribution of uncorrelated YSR levels in a narrow band, $W \ll 2 E_{\mathrm{YSR}}$, and ensemble-averaging the conductivity, we find [27]

$$
\begin{aligned}
\left\langle\frac{\sigma(\omega)}{\sigma_{n}}\right\rangle= & \frac{\bar{F}(\{\mathbf{S}(\mathbf{r})\})}{\hbar \omega} \frac{2 \alpha^{4}}{3\left(1+\alpha^{2}\right)^{2}} \frac{n^{2}}{\hbar \pi v^{2} \Delta}(w / \xi)^{3-d} \\
& \times \frac{\xi}{l} \frac{1}{2 W \Delta} g\left(\hbar \omega-2 \bar{E}_{\mathrm{YSR}}, W\right),
\end{aligned}
$$

where $\bar{\alpha}$ is the mean value of $\alpha_{i}$ and $\bar{E}_{\mathrm{YSR}}$ the average YSR energy. The normalized distribution function $g(\omega, W)=$ $2(1-|\omega| / W)^{3} \Theta(W-|\omega|) / W$ (specific to a uniform YSR band) characterizes the energy dependence of the absorption and has a peak of width $W$. We normalize the conductivity by its normal-state value $\sigma_{n}=2 e^{2} v \frac{1}{2} v_{F} l$, where $l$ is the electron mean free path from nonmagnetic impurities; Eq. (10) is valid in the clean limit $l \gg \xi$. The dirty limit $l \ll \xi$ is obtained by replacing $\xi \rightarrow l$ in the second line of Eq. (10) [32,41].

In the high-density regime, $n \xi_{\text {YSR }}^{3} \gg 1$, the YSR states will strongly hybridize. The conductivity in this case is derived in Ref. [32]. At a qualitative level, the high-density limit can be obtained from Eq. (10) by replacing $\xi$ (in the second line) by an effective mean free path arising from magnetic impurities, $\xi \rightarrow 1 /\left(n \xi^{2}\right)$. When the above conductivity is integrated over the subgap states, one naturally recovers Eq. (1).

Finally, we now estimate the achievable strength of the dressed interactions induced by an off-resonant microwave field. The relevant energy levels and matrix element are depicted in Fig. 1(b). At leading order, one finds that the radiation-assisted YSR pair creation results in an effective spin-spin interaction originating from a spin-dependent AC stark shift to the ground-state energy,

$$
H_{\mathrm{eff}}=\delta E_{\mathrm{GS}}=\frac{\left|\mathcal{M}_{\mathrm{YSR}}\right|^{2}}{\omega-2 E_{\mathrm{YSR}}}=J_{\mathrm{YSR}}^{\mathrm{ind}} \mathbf{S}_{1} \cdot \mathbf{S}_{2},
$$

where we have neglected a spin-independent overall shift. To compare the relative strength of this dressed interaction with the RKKY component, we use Eq. (8) and the expression for the RKKY interaction from Ref. [17], resulting [27] in the estimate presented in Eq. (2).

\section{CONCLUSION}

In summary, we have shown that the electronic subgap states hosted by magnetic impurities in a superconductor provide a way to access magnetic order. This opens the possibility to probe and control metallic spin glass physics 
in a superconducting narrow-bridge junction by using microwave driving [Fig. 1(a)]. Keeping the transverse size $w$ of the bridge thinner than the London length ensures that the supercurrent is approximately uniform and couples to the magnetic moments in the full volume. Unlike conventional Andreev bound states, the YSR levels are insensitive to a static phase difference across the junction which provides a simple way to distinguish the respective contributions to the dissipative conductivity. Looking forward, our work also opens the door to an intriguing quantum information platform where magnetic impurities in a superconducting host play the role of quantum memories, while microwave driving can lead to on-demand long-range gates [42]. Here, the absence of a power-law decay in the radiation matrix element could enable all-to-all connectivity between qubits as well as multibody interactions, both of which are important for reducing the gate depth of certain quantum algorithms $[43,44]$ and realizing frustrated long-range spin models $[45,46]$.

\section{ACKNOWLEDGMENTS}

We gratefully acknowledge the insights of and discussions with K. Murch, I. Siddiqi, and T. Ojanen. The authors would particularly like to thank G. Zarand for previous collaborations on related work and for pointing out the impact of reflection symmetry. This work was supported by the U.S. Department of Energy, Office of Basic Energy Sciences, Materials Sciences and Engineering Division, under Contract No. DE-AC02-05-CH11231 within the HighCoherence Multilayer Superconducting Structures for Large Scale Qubit Integration and Photonic Transduction Program (QISLBNL), and by the David and Lucile Packard Foundation and the Sloan Foundation. J.D.S. acknowledges support from the NSF-DMR1555135 (CAREER). E.A.D. acknowledges support from the Harvard-MIT CUA, the AFOSR-MURI Photonic Quantum Matter (award FA95501610323), and the DARPA DRINQS program (award D18AC00014). L.I.G. acknowledges support from the NSF DMR Grant No. 1603243.
[1] K. H. Fischer and J. A. Hertz, Spin Glasses, Cambridge Studies in Magnetism (Cambridge University, New York, 1991).

[2] M. A. Ruderman and C. Kittel, Phys. Rev. 96, 99 (1954).

[3] T. Kasuya, Prog. Theor. Phys. 16, 45 (1956).

[4] K. Yosida, Phys. Rev. 106, 893 (1957).

[5] S. F. Edwards and P. W. Anderson, J. Phys. F: Metal Phys. 5, 965 (1975)

[6] G. Parisi, J. Phys. A: Math. Gen. 13, 1101 (1980).

[7] D. Sherrington and S. Kirkpatrick, Phys. Rev. Lett. 35, 1792 (1975).

[8] D. J. Thouless, P. W. Anderson, and R. G. Palmer, Philos. Mag. 35, 593 (1977).

[9] J. R. L. de Almeida and D. J. Thouless, J. Phys. A: Math. Gen. 11, 983 (1978).

[10] V. Cannella and J. A. Mydosh, Phys. Rev. B 6, 4220 (1972).

[11] V. Cannella, J. A. Mydosh, and J. I. Budnick, J. Appl. Phys. 42, 1689 (1971).

[12] V. Cannella and J. A. Mydosh, AIP Conf. Proc. 10, 785 (1973).

[13] T. Capron, A. Perrat-Mabilon, C. Peaucelle, T. Meunier, D. Carpentier, L. P. Lévy, C. Bäuerle, and L. Saminadayar, Europhys. Lett. 93, 27001 (2011).

[14] P. G. N. de Vegvar, L. P. Lévy, and T. A. Fulton, Phys. Rev. Lett. 66, 2380 (1991).

[15] T. Capron, G. Forestier, A. Perrat-Mabilon, C. Peaucelle, T. Meunier, C. Bäuerle, L. P. Lévy, D. Carpentier, and L. Saminadayar, Phys. Rev. Lett. 111, 187203 (2013).

[16] M. H. Devoret and R. J. Schoelkopf, Science 339, 1169 (2013).

[17] N. Y. Yao, L. I. Glazman, E. A. Demler, M. D. Lukin, and J. D. Sau, Phys. Rev. Lett. 113, 087202 (2014).

[18] L. Yu, Acta Phys. Sin. 21, 75 (1965).

[19] H. Shiba, Prog. Theor. Phys. 40, 435 (1968).

[20] A. I. Rusinov, Sov. J. Exp. Theor. Phys. Lett. 9, 85 (1969).

[21] M. Ruby, Y. Peng, F. von Oppen, B. W. Heinrich, and K. J. Franke, Phys. Rev. Lett. 117, 186801 (2016).

[22] B. W. Heinrich, J. I. Pascual, and K. J. Franke, Prog. Surf. Sci. 93, 1 (2018).
[23] M. Ruby, F. Pientka, Y. Peng, F. von Oppen, B. W. Heinrich, and K. J. Franke, Phys. Rev. Lett. 115, 087001 (2015).

[24] M. Ruby, B. W. Heinrich, Y. Peng, F. von Oppen, and K. J. Franke, Phys. Rev. Lett. 120, 156803 (2018).

[25] J. O. Island, R. Gaudenzi, J. de Bruijckere, E. Burzurí, C. Franco, M. Mas-Torrent, C. Rovira, J. Veciana, T. M. Klapwijk, R. Aguado, and H. S. J. van der Zant, Phys. Rev. Lett. 118, 117001 (2017).

[26] L. Farinacci, G. Ahmadi, G. Reecht, M. Ruby, N. Bogdanoff, O. Peters, B. W. Heinrich, F. von Oppen, and K. J. Franke, Phys. Rev. Lett. 121, 196803 (2018).

[27] A single YSR state, however, may affect a single-photon state in a cavity surrounding the host superconductor, see Ref. [47].

[28] A. Yazdani, B. A. Jones, C. P. Lutz, M. F. Crommie, and D. M. Eigler, Science 275, 1767 (1997).

[29] S.-H. Ji, T. Zhang, Y.-S. Fu, X. Chen, X.-C. Ma, J. Li, W.-H. Duan, J.-F. Jia, and Q.-K. Xue, Phys. Rev. Lett. 100, 226801 (2008).

[30] L. Cornils, A. Kamlapure, L. Zhou, S. Pradhan, A. A Khajetoorians, J. Fransson, J. Wiebe, and R. Wiesendanger, Phys. Rev. Lett. 119, 197002 (2017).

[31] S. Kezilebieke, R. Žitko, M. Dvorak, T. Ojanen, and P. Liljeroth, Nano Lett. 19, 4614 (2019).

[32] Y. V. Fominov, M. Houzet, and L. I. Glazman, Phys. Rev. B 84, 224517 (2011).

[33] S. Kezilebieke, M. Dvorak, T. Ojanen, and P. Liljeroth, Nano Lett. 18, 2311 (2018).

[34] D.-J. Choi, C. G. Fernández, E. Herrera, C. Rubio-Verdú, M. M. Ugeda, I. Guillamón, H. Suderow, J. I. Pascual, and N. Lorente, Phys. Rev. Lett. 120, 167001 (2018).

[35] C. Cohen-Tannoudji, J. Dupont-Roc, and G. Grynberg, AtomPhoton Interactions: Basic Processes and Applications (WileyInterscience publication, New York, 1992).

[36] See Supplemental Material at http://link.aps.org/supplemental/ 10.1103/PhysRevResearch.1.033091 for a derivation of the pair transition matrix element, the integrated conductivity in the clean dilute limit, and calculation of the ratio $J_{\mathrm{YSR}}^{\text {ind }} / J_{\mathrm{RKKY}}$. 
[37] E. M. Levenson-Falk, F. Kos, R. Vijay, L. Glazman, and I. Siddiqi, Phys. Rev. Lett. 112, 047002 (2014).

[38] C. Janvier, L. Tosi, L. Bretheau, Ç. Girit, M. Stern, P. Bertet, P. Joyez, D. Vion, D. Esteve, M. Goffman et al., Science 349, 1199 (2015).

[39] M. Hays, G. de Lange, K. Serniak, D. J. van Woerkom, D. Bouman, P. Krogstrup, J. Nygård, A. Geresdi, and M. H. Devoret, Phys. Rev. Lett. 121, 047001 (2018).

[40] L. Tosi, C. Metzger, M. F. Goffman, C. Urbina, H. Pothier, S. Park, A. L. Yeyati, J. Nygård, and P. Krogstrup, Phys. Rev. X 9 , 011010 (2019).

[41] T. Kiendl, F. von Oppen, and P. W. Brouwer, Phys. Rev. B 96, 134501 (2017).
[42] J. Levy, Phys. Rev. Lett. 89, 147902 (2002).

[43] E. Bernstein and U. Vazirani, SIAM J. Comput. 26, 1411 (1997).

[44] W. van Dam, S. Hallgren, and L. Ip, SIAM J. Comput. 36, 763 (2006).

[45] S. Sachdev and J. Ye, Phys. Rev. Lett. 70, 3339 (1993).

[46] A. Kitaev, KITP Strings Seminar and Entanglement Program, 2015 (unpublished).

[47] R. Chirla, A. Manolescu, and C. P. Moca, Phys. Rev. B 93 , 155110 (2016).

Correction: Incorrect information in a support statement in the Acknowledgments has been fixed. 